\title{
Local dissipation limits the dynamics of impacting droplets on smooth and rough substrates
}

\author{
Yuli Wang, ${ }^{1,2, *}$ Gustav Amberg,,${ }^{1,3}$ and Andreas Carlson ${ }^{2, \dagger}$ \\ ${ }^{1}$ Department of Mechanics, Royal Institute of Technology, 10044 Stockholm, Sweden \\ ${ }^{2}$ Department of Mathematics, Mechanics Division, University of Oslo, 0851 Oslo, Norway \\ ${ }^{3}$ Södertörn University, 14189 Huddinge, Sweden \\ (Received 24 November 2016; published 28 March 2017)
}

\begin{abstract}
A droplet that impacts onto a solid substrate deforms in a complex dynamics. To extract the principal mechanisms that dominate this dynamics, we deploy numerical simulations based on the phase field method. Direct comparison with experiments suggests that a dissipation local to the contact line limits the droplet spreading dynamics and its scaled maximum spreading radius $\beta_{\max }$. By assuming linear response through a drag force at the contact line, our simulations rationalize experimental observations for droplet impact on both smooth and rough substrates, measured through a single contact line friction parameter $\mu_{f}$. Moreover, our analysis shows that dissipation at the contact line can limit the dynamics and we describe $\beta_{\max }$ by the scaling law $\beta_{\max } \sim\left(\operatorname{Re} \mu_{1} / \mu_{f}\right)^{1 / 2}$ that is a function of the droplet viscosity $\left(\mu_{1}\right)$ and its Reynolds number $(\mathrm{Re})$.
\end{abstract}

DOI: 10.1103/PhysRevFluids.2.033602

\section{INTRODUCTION}

Impact of liquid droplets onto a solid substrate is essential to applications such as spray coating [1], ink-jet printing [2], additive manufacturing [3] and pesticide deposition [4]. Upon impact with the substrate, the droplet deforms in a complex dynamics, where a gas film can become trapped underneath the droplet [5-12] and as it spreads creates a splash by droplet ejection at the tip of its spreading front [13-17]. The droplet deformation and spreading are typically driven by its inertia and hindered by viscous and surface tension forces. Two nondimensional numbers are particularly relevant to characterize the dynamics, which is the Reynolds number $\operatorname{Re}=\rho_{1} V_{i} 2 R / \mu_{1}$ giving the ratio between inertia and viscous forces and the Weber number $\mathrm{We}=\rho_{1} V_{i}^{2} 2 R / \sigma$ which gives the ratio between inertia and surface tension forces. $\sigma$ is the surface tension coefficient of the gas-liquid, $\rho_{1}$ is the liquid density, $\mu_{1}$ is the liquid viscosity, and $V_{i}$ is the droplet impact speed. Besides inertia, viscosity, and surface tension, we hypothesize and show that a dissipation local to the contact line can limit the droplet dynamics on both smooth and rough substrates.

One parameter that describes the droplet impact dynamics and is typically quantified as the spreading factor $\beta(t)=r(t) / R$, where $r(t)$ is the droplet spreading radius, $R$ is the initial droplet radius, and $\beta_{\max }=\max [\beta(t)]$; see Fig. 1 . Two primary regimes have been identified to describe $\beta_{\max }$ : an inertia-viscous regime where $\beta_{\max } \sim \operatorname{Re}^{\frac{1}{5}}[18,19]$ and an inertia-capillary regime where $\beta_{\max } \sim \mathrm{We}^{\frac{1}{2}}[20,21]$. A single law has been derived to connect these two regimes, $\beta_{\max } \operatorname{Re}^{-\frac{1}{5}} \sim$ $f\left(\mathrm{WeRe}^{-\frac{2}{5}}\right)$ [22], which has rationalized experiments for a wide range of Re and We numbers [23]. Other scaling laws for $\beta_{\max }$ with different exponents for $\mathrm{Re}$ and We have been proposed [24-30], which include additional effects such as the substrate wettability. A detailed description of these different scaling laws can be found in the recent review by Josserand and Thoroddsen [31]. However, none of these scaling laws describe $\beta_{\max }$ at low impact speeds as they predict $\beta_{\max } \rightarrow 0$ for $V_{i} \rightarrow 0$, which is not true for any case with an equilibrium contact angle $\theta_{\mathrm{e}}<180^{\circ}$. To mitigate this artifact, the maximum spreading radius $\beta_{0}$ for $V_{i}=0$ has been included into the analysis

\footnotetext{
*yuli@mech.kth.se

†acarlson@math.uio.no
} 


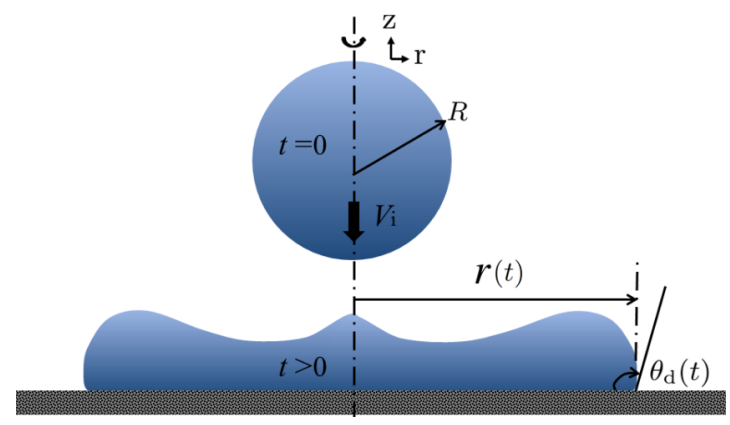

FIG. 1. Sketch of the axisymmetric computational domain and the droplet's initial condition with $R$ the initial droplet radius and $V_{i}$ the impact speed. The spreading radius $r(t)$ and the apparent dynamic contact angle $\theta_{\mathrm{d}}(t)$ are illustrated as the droplet has spread onto the solid substrate.

$\sqrt{\beta_{\max }^{2}-\beta_{0}^{2}}=\mathrm{Re}^{\frac{1}{5}} \mathrm{We}^{\frac{1}{2}} /\left(A+\mathrm{We}^{\frac{1}{2}}\right)$ that agrees favorably with experimental data for both low and high impact speeds [32], where $A$ is an ad hoc fitting parameter.

Substrate roughness is another parameter that can influence the droplet impact dynamics [33-37]. Droplet impact on regular microtextured substrates [33,34,38] shows that $\beta_{\max }$ is influenced by the substrate topography. Even a substrate with small aspect ratio roughness hinders droplet spreading [35], although the effect becomes less pronounced.

In this work we focus on describing $\beta(t)$ and $\beta_{\max }$ in the regime of nonsplashing droplets [31,39], i.e., small and intermediate impact speed. We show that as in a spontaneous droplet spreading process [40,41], a detailed description of the physical processes at the contact line must be included to accurately describe the interface dynamics. Numerical experiments based on the phase field method and the Navier-Stokes equations show that friction local to the contact line limits the dynamics and generates a significant dissipation. We treat the contact line friction parameter $\mu_{f}$ as a material property for each combination of air, liquid, and solid, which should be independent of the impact speed. We determine the magnitude of $\mu_{f}$ by directly comparing the numerical simulations with several independent experiments [23,25,32,34,35]. Our assumption of linear response through a Stokes-like drag at the contact line shows that the simulations can accurately reproduce experimental observations. We further extend our analysis to rough substrates and rationalize the differences in the dynamics compared with smooth substrates. Finally, we show that the regime where the principal dissipation is local to the contact line is described by a scaling law based on the contact line friction parameter $\mu_{f}$.

\section{A. Models and methods}

We describe the multiphase system by using the phase field method [42], which considers the two binary phases (gas and liquid) as a mixture. The mathematical model is composed of the Cahn-Hilliard equation [43] Eqs. (1) and (2), which are coupled with the Naiver-Stokes equations (3) and (4) for an incompressible fluid flow [42]:

$$
\begin{gathered}
\frac{\partial C}{\partial t}+\mathbf{u} \cdot \nabla C=\gamma \nabla^{2} \phi \\
\phi=-\frac{3}{2 \sqrt{2}} \sigma\left[\epsilon \nabla^{2} C-\frac{\delta \psi(C)}{\delta C}\right], \\
\rho(C)\left[\frac{\partial \mathbf{u}}{\partial t}+(\mathbf{u} \cdot \nabla) \mathbf{u}\right]=-\nabla P+\nabla \cdot\left[\mu(C)[\nabla \mathbf{u}+(\nabla \mathbf{u})]^{T}\right]+\phi \nabla C, \\
\nabla \cdot \mathbf{u}=0 .
\end{gathered}
$$


TABLE I. Simulated material properties and droplet impact conditions on substrates with different equilibrium contact angles $\left(\theta_{\mathrm{e}}\left([\text { steel, stainless steel, aluminium, grooved stainless } s t e e l]_{\text {air-water }}\right)=\right.$ $\left[61^{\circ}, 90^{\circ}, 94^{\circ}, 130^{\circ}\right]$ and $\left.\theta_{\mathrm{e}}\left([\text { steel }]_{\text {air-glycerol } / \text { water }}\right)=\left[52^{\circ}\right]\right)$.

\begin{tabular}{lcccccccc}
\hline \hline & $\rho_{l}\left(\mathrm{~kg} \mathrm{~m}^{-3}\right)$ & $\mu_{1}(\mathrm{Pas})$ & $\sigma\left(\mathrm{N} \mathrm{m}^{-1}\right)$ & $R(\mathrm{~mm})$ & $V_{i}(\mathrm{~m} / \mathrm{s})$ & $\theta_{e}\left(^{\circ}\right)$ & $\mathrm{Re}$ & $\mathrm{We}$ \\
\hline Water & 1000 & 0.001 & 0.073 & 1 & $0.28-4.85$ & $61,90,94,130$ & $320-10^{4}$ & $0.6-664$ \\
Glycerol-water & 1158 & 0.01 & 0.068 & 0.92 & $0.19-9.28$ & 52 & $40-1956$ & $1-2653$ \\
\hline \hline
\end{tabular}

$C=C(r, z, t)$ is an order parameter that varies smoothly from $C=-1$ (gas) to $C=1$ (liquid) between the two immiscible phases and $|C|<1$ indicates that the interfacial region that has a finite thickness $\epsilon . \phi=\phi(r, z, t)=\delta F(r, z, t) / \delta C$ is the chemical potential, given by the variation of the systems postulated free energy $F(r, z, t)$ that has an interfacial $\left(\frac{\sigma \epsilon}{2}|\nabla C|^{2}\right)$ and bulk free energy term $\left[\frac{\sigma}{\epsilon} \psi(C)=\frac{\sigma}{4 \epsilon}(C+1)^{2}(C-1)^{2}\right]$. The free energy is required to reduce with time, i.e., $\gamma>0$ and $\gamma$ is the mobility factor that controls the interfacial diffusion. $\mathbf{u}=\mathbf{u}(r, z, t)$ is the velocity, $P=P(r, z, t)$ is the pressure, whereas the density $\rho(C)=(1+C) \rho_{\mathrm{l}} / 2+(1-C) \rho_{\mathrm{g}} / 2$ and the viscosity $\mu(C)=(1+C) \mu_{1} / 2+(1-C) \mu_{\mathrm{g}} / 2$ are interpreted as function of $C$. The air surrounding the droplet is assumed at atmospheric pressure with a density $\rho_{\mathrm{g}}=1.23 \mathrm{~kg} / \mathrm{m}^{3}$ and a viscosity $\mu_{\mathrm{g}}=1.81 \times 10^{-5}$ Pa s. The material properties of the droplet, along with the impact speeds, equilibrium contact angles, and range of simulated Re numbers and We numbers are listed in Table I.

All simulations are performed with a no-slip boundary condition for the velocities at the solid substrate $(\mathbf{u}=0)$ and all other boundaries are assumed to be in contact with ambient air at constant pressure $(P=0)$ and with no flux of the chemical potential $(\nabla \phi \cdot \mathbf{n}=0$ with $\mathbf{n}$ as the boundary normal). To model the contact line dynamics we use the nonequilibrium boundary condition [44],

$$
\frac{2 \sqrt{2}}{3} \sigma \epsilon \nabla C \cdot \mathrm{n}+\sigma \cos \left(\theta_{\mathrm{e}}\right) g^{\prime}(C)=-\mu_{f} \epsilon \frac{\partial C}{\partial t},
$$

where $\mu_{f}$ is interpreted as a friction factor at the contact line and $g(C)=-2 / 4-3 / 4 C+1 / 4 C^{3}$ gives the transition from dry (gas-solid) to wet (liquid-solid) substrate surface tension that is derived directly from the double-well function in the bulk free energy $\psi(C)$. Interfacial diffusion ameliorates the stress singularity at the contact line and in the sharp interface limit $[45,46]$ the phase field model becomes independent of the interface thickness [40], which in practice is chosen to be larger than what can be argued physically for simulations of macroscopic flows.

We use the following scaling: $\mu^{*}(C)=\mu(C) / \mu_{1}, \rho^{*}(C)=\rho(C) / \rho_{1}, \mathbf{u}^{*}=\mathbf{u} / V_{i}, t^{*}=$ $t V_{i} / R, P^{*}=P R /\left(\mu_{1} V_{i}\right), \phi^{*}=\phi 2 \sqrt{2} \epsilon /(3 \sigma)$ to make Eqs. (1)-(5) nondimensional, where the superscript $*$ denotes nondimensional variables. In addition to the Reynolds number $(\mathrm{Re})$ four nondimensional numbers appear in the equations: the capillary number $\mathrm{Ca}=\mathrm{We} / \mathrm{Re}=V_{i} \mu_{1} / \sigma$ gives the ratio of the viscous force to the surface tension force, the ratio between the dynamic viscosity, and the contact line friction parameter $\eta=\mu / \mu_{f}$, the Cahn number $\mathrm{Cn}=\epsilon / R=0.005$ gives the ratio of the interface thickness to the droplet radius, and the Péclet number $\mathrm{Pe}=2 \sqrt{2} V_{i} \in R /(3 \sigma \gamma)=100$ gives the ratio of advection to diffusion. Both $\mathrm{Cn}$ and $\mathrm{Pe}$ are fixed in all of our simulations such that the results satisfy the sharp interface criterion $[45,46]$. The contour line $C=0$ is interpreted as the droplet interface and used to extract $\beta(t), \beta_{\max }$, and $\theta_{\mathrm{d}}(t)$.

The numerical simulations are performed with FemLego [47], a symbolic finite-element toolbox that solves partial differential equations. All simulations are performed in an axisymmetric coordinate system where the domain extends $10 R$ in the $r$ direction and $5 R$ in the $z$ direction. An adaptive mesh refinement method is used to enable a high resolution of the interface with a minimum mesh size of $\Delta r \approx \Delta z \approx 0.001 R$, which resolves the interface with $\mathrm{Cn} / \Delta r \approx 5$ cells. The short and long time scales require us to closely monitor the time stepping, which is adapted during the simulations to ensure that the Courant-Friedrichs-Lewy condition $<0.1$. The droplet's center of mass is initialized 
at a height $z=1.5 R$ from the solid substrate and the dynamic contact angle $\theta_{\mathrm{d}}(t)$ is measured at a height of $z=100 \mu \mathrm{m}$ using linear interpolation along $C=0$ similar to the method used to in the experimental analysis that we are directly comparing against [32].

The phase field method has previously been used with success to simulate droplet impact dynamics $[48,49]$ to quantify the early spreading and bubble entrapment, in accordance with experimental observations [50]. However, none of these account for a dynamic contact angle treatment in Eq. (5) with $\mu_{f}>0$ or quantify the maximum spreading radius of the droplet, which we will show are two intertwined processes needed to rationalize the impact dynamics of droplets on smooth and rough solid substrates.

\section{RESULTS AND DISCUSSION}

\section{A. Droplet impact on smooth substrate}

Phase field simulations of droplets that spread onto a solid substrate has previously been shown to compare favorably with experiments and classical theory for viscous spreading [51], as well as spreading dynamics where the apparent contact angle is so far from equilibrium that it requires $\mu_{f}>0$ in Eq. (5) [41]. The mathematical form of Eq. (5) comes from the assumption of linear response with a reduction of the free energy in time and can be interpreted as a Stokes-like drag at the contact line. We interpret this wall-interface friction parameter $\mu_{f}$ as a physical property that depends on the combination of the gas, liquid, and solid. We hypothesize that parts of the parameter space that compose the droplet impact dynamics can only be described with an accurate local treatment of the dynamic contact angle through $\mu_{f}$.

Since $\mu_{f}$ is not known a priori we determine its magnitude by directly comparing simulations with experiments [25,32], where $\mu_{f}$ is identified as the best match with $\beta(t)$ (see Fig. 2). Our simulations of droplets of water and glycerol-water mixture show that $\mu_{f}$ clearly affects the spreading dynamics as well as the shape of the droplet. For water droplets on steel $\mu_{f} \sim 0.52 \mathrm{~Pa}$ s, while increasing its viscosity by introducing glycerol $\left(\mu_{1}=0.01 \mathrm{~Pa} \mathrm{~s}\right)$ also increases $\mu_{f} \sim 0.72 \mathrm{~Pa}$. These magnitudes for $\mu_{f}$ are in accordance with previous measurements on spontaneous spreading droplets $\left(V_{i}=0\right)$ [41]. Our simulations clearly show that $\mu_{f}$ controls the time scale for $\theta_{\mathrm{d}}(t)$ to approach the equilibrium angle, where $\theta_{\mathrm{d}}(t)$ is the droplet's apparent contact angle; see Figs. 2(b) and 2(d). It is noteworthy that the assumption of local equilibrium, i.e., equilibrium contact angle, overpredicts the spreading factor $r(t)$ and its maximum $r_{\max }=\max [r(t)]$ or in nondimensional units $\beta_{\max }=$ $\max [\beta(t)]$, for both liquids. Thus, to obtain agreement between the simulations and experiments, we need to account for dissipation at the contact line and the local equilibrium assumption fails to capture the spatiotemporal droplet dynamics; see Figs. 2(b) and 2(d). An alternative and simpler way to extract $\mu_{f}$ would be to compare droplet-spreading experiments with the analytical relation for the contact line speed [52] derived from Eq. (5), but these data are, to the best of our knowledge, not available for the given liquid-gas-substrate combination.

After determining $\mu_{f}$ (Table II) from an experiment for one impact speed $V_{i}$, we now assume $\mu_{f}$ to be a constant material parameter that must be independent of $V_{i}$. We challenge our hypothesis that $\mu_{f}$ is unique for a specific air-liquid-solid combination by directly comparing our simulations with experiments [32,35] for different $V_{i}$. It is clear that as we increase $V_{i}$ the droplets aspect ratio, i.e., maximum height divided by $\beta_{\max }$, decreases at $\beta(t)=\beta_{\max }$. The simulated droplet shapes are in very good agreement with the experiments at $\beta_{\max }$, where the difference in profiles [Figs. 3(a), $3(\mathrm{c})$, and 3(d)] is caused by the experimental side-view photos. Since the simulations show a slice through the droplet, they represent its actual shape. The dash-dotted lines in panels to the right in Fig. 3 illustrate how the shape of the droplet would look if we instead would have made a side-view image. Our simulations also capture the entrapment of an air bubble at the symmetry axis at the wall, as seen in Figs. 3(a)-3(c).

It is clear that $\mu_{f}$ needs to be determined individually for each air-liquid-substrate combination. A too small value for $\mu_{f}$ causes an overprediction of $\beta_{\max }$, while a too large value for $\mu_{f}$ causes an underprediction of $\beta_{\max }$; see Fig. 4. We want to highlight that the value for $\mu_{f}$ determined from 
(a)

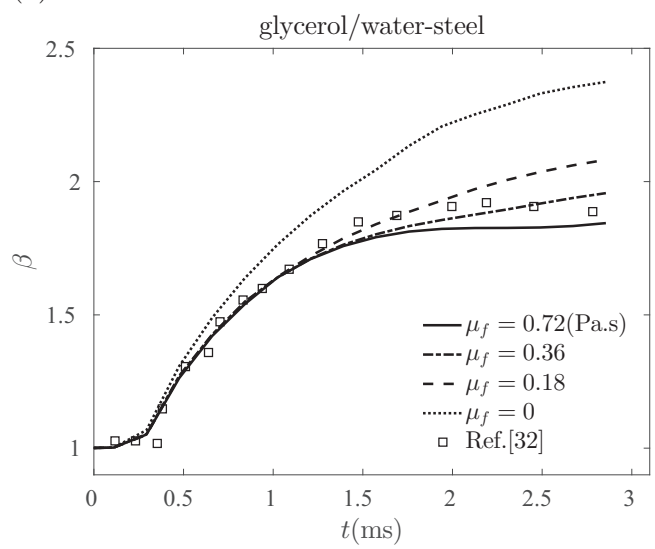

(c)

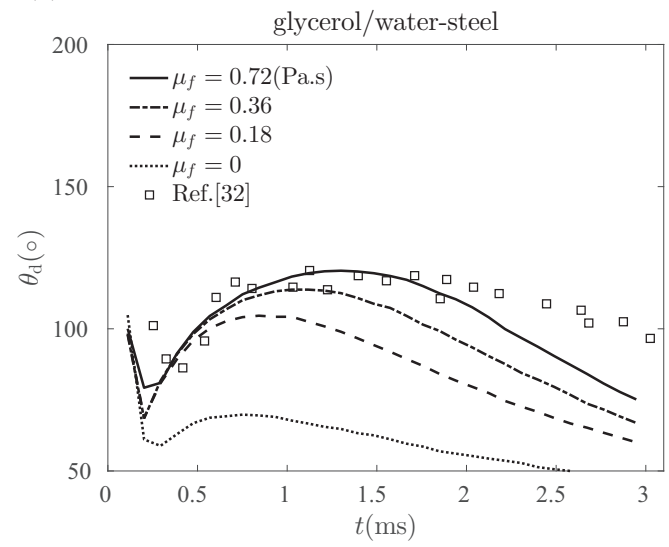

(b)

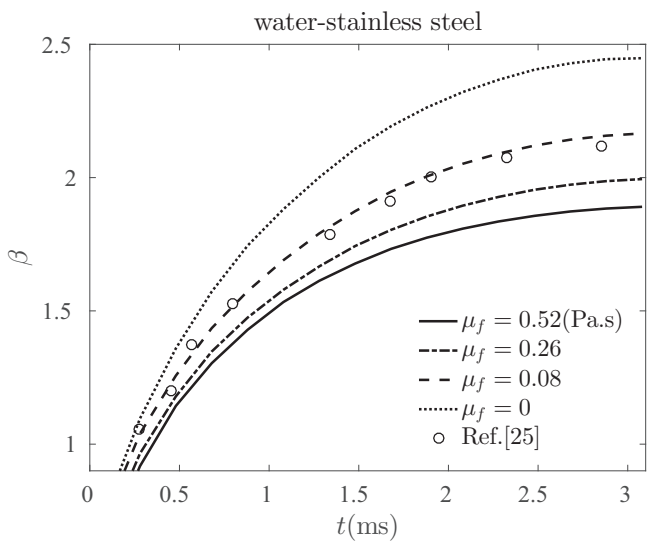

(d)

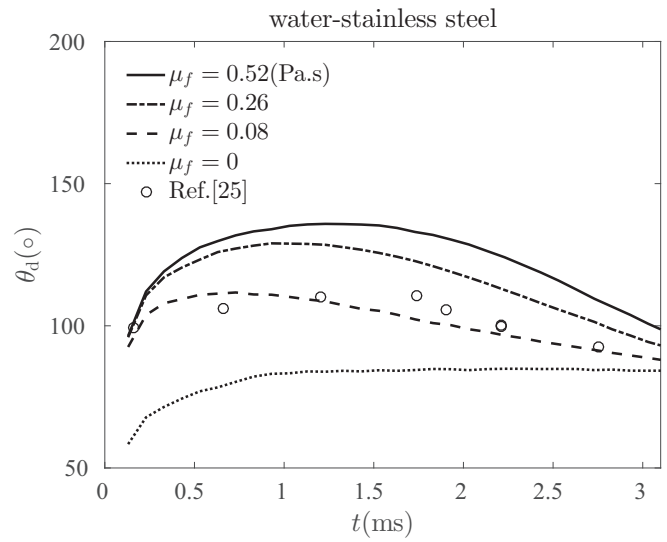

FIG. 2. Comparison of experimental data (markers) and numerical simulations (lines) for a droplet impacting onto smooth substrates with a speed $V_{i}=1 \mathrm{~m} / \mathrm{s}$. (a), (b) $\beta(t)$ and (c), (d) $\theta_{\mathrm{d}}(t)$ as a function of the viscosity $\left(\mu_{1}\right)$ and the contact line friction parameter $\left(\mu_{f}\right)$, using the same definition of $\beta(t)$ as reported in experiments $[32,35]$.

a single experiment is also the best fit for a range of impact speeds $V_{i}$ and shows that $\mu_{f}$ is not a function of $V_{i}$.

\section{B. Droplet impact on textured substrates}

Another parameter that can influence droplet spreading upon impact is the substrate topography $[34,38]$. For spontaneous spreading of droplets $\left(V_{i}=0\right)$ the friction factor $\mu_{f}$ has already been shown

TABLE II. Measurement of $\mu_{f}$ as air-liquid-solid combinations.

\begin{tabular}{lccc}
\hline \hline Liquid-substrate & $\theta_{e}\left({ }^{\circ}\right)$ & $\mu_{f}(\mathrm{~Pa} \mathrm{~s})$ & Experiments \\
\hline Glycerol/water-steel & 52 & 0.72 & Ref. [32] \\
Water-stainless steel & 90 & 0.08 & Ref. [25] \\
Water-steel & 61 & 0.52 & Ref. [32] \\
Water-aluminium & 94 & 0.08 & Ref. [35] \\
\hline \hline
\end{tabular}


(a)

(b)

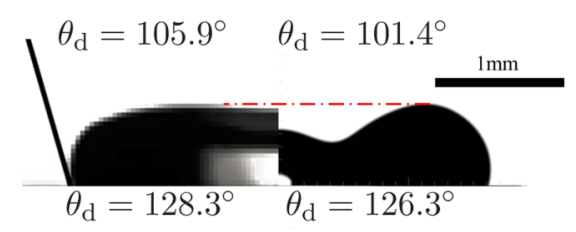

(c)

(d)
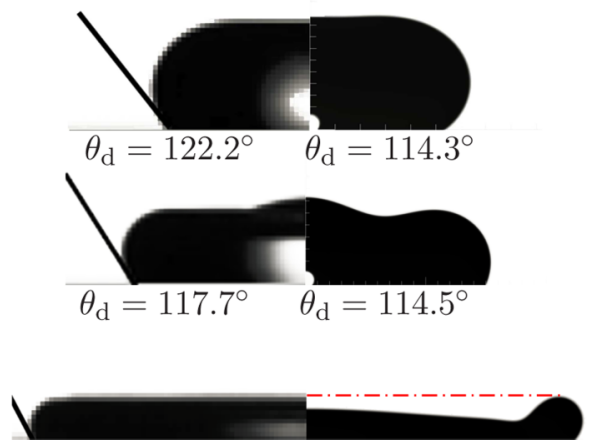

FIG. 3. Comparison of the experimental [32] (the left half, their Fig. 2) and numerical (the right half) droplet shape as it impacts onto a steel substrate. (a) A water droplet ( $1 \mathrm{mPa} \mathrm{s}$ ) with $\mu_{f}=0.52 \mathrm{~Pa}$ and $V_{i}=0.57 \mathrm{~m} / \mathrm{s}$. (b)-(d) A glycerol-water droplet (10 mPa s) with $\mu_{f}=0.72 \mathrm{~Pa} \mathrm{~s}$ and (b) $V_{i}=0.28 \mathrm{~m} / \mathrm{s}$, (c) $V_{i}=0.6 \mathrm{~m} / \mathrm{s}$, and (d) $V_{i}=1.86 \mathrm{~m} / \mathrm{s}$.

to rationalize spreading dynamics on rough substrates [53] where the magnitude of $\mu_{f}$ depends on the substrate topography which is classified by the roughness factor $S$ i.e. the ratio of the projected substrate area to the real substrate area where a smooth substrate has $S=1$. To test if our description of the contact line dynamics can provide a universal framework that can effectively bridge impact dynamics on smooth and rough substrates, we test the relation for the effective contact line friction parameter $\mu_{\text {eff }} \sim S \mu_{f}$ [53], having already estimated the value for $\mu_{f}$ for the smooth substrate. In the experiments we compare against, the substrate has a quasi-two-dimensional structure [34] (see Fig. 5) and its geometry gives the following roughness factor $S=\frac{b+w-2 d \cos (\alpha)+2 d / \sin (\alpha)}{b+w}$, which is the ratio of the real area to projected area of the substrate. $b, w, d$, and $\alpha$ are geometric parameters describing the grooved substrate of this particular substrate that we compare our simulations with; see the inset in Fig. 5. If the contact line friction parameter $\mu_{f}$ is known for the corresponding flat substrate, the effective friction $\mu_{\text {eff }}$ can easily be determined once the geometry of the microtextured substrate is known.

We compare our simulations with experiments on substrates that have grooves along one direction with different aspect ratios. In Fig. 5 the effective contact line friction parameter $\mu_{\text {eff }}$ is determined by matching the experimental data for the TS11 substrate [34] with a roughness factor $S_{11}=1.27$, where we test the relation $\mu_{\mathrm{eff}} \sim S \mu_{f}$ [53] for the substrates $\operatorname{TS} 140\left(S_{140}=1.79\right)$ and $\operatorname{TS} 220\left(S_{220}=2.45\right)$ [34]. Although our assumption of axial symmetry is slightly violated in the experiments, the linear relationship between $S$ and $\mu_{\text {eff }}$ rationalizes $\beta_{\max }$ for the spreading perpendicular to the grooves. This is also believed to be the primary cause for the difference in $\mu_{f}$ found for a water droplet impacting on the smooth $\left(\mu_{f}=0.08 \mathrm{Pas}\right)$ and textured $\left(\mu_{f}=0.28 \mathrm{~Pa} \mathrm{~s}\right)$ stainless steel substrate.

\section{Energy budget}

Our results show that the local interface-wall contact line friction can affect the droplet impact dynamics, and we want next to determine its dissipative contribution and compare it against the other primary contributions in the energy budget. To do this, we extract the different rates of energy and dissipations, where the principal contributions are the rate of change of kinetic energy $R_{\rho}=\frac{1}{2} \int_{\Omega} \partial\left[\rho(C) \mathbf{u}^{2}\right] / \partial t d \Omega$, the rate of viscous dissipation $R_{\mu}=\int_{\Omega} \mu(C)\left(\nabla \mathbf{u}+\nabla \mathbf{u}^{\mathbf{T}}\right): \nabla \mathbf{u} d \Omega$, 

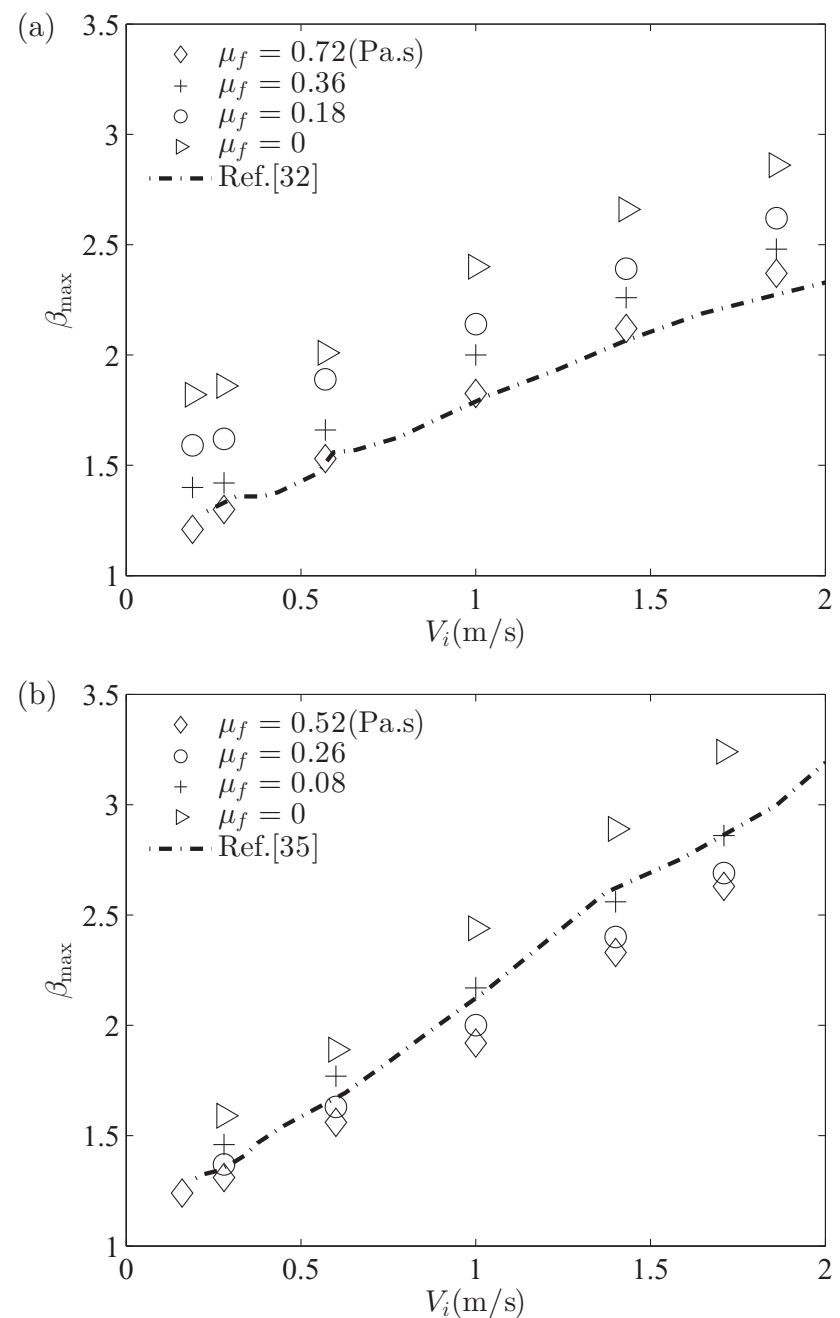

FIG. 4. The maximum spreading factor $\beta_{\max }=\max [\beta(t)]$ as a function of impact speed $V_{i}$ and $\mu_{f}$. (a) Droplets with a glycerol-water mixture impacting onto a steel substrate, where the dashed line is interpolated experimental data [32] (their Fig. 4). (b) Water droplets impacting onto an aluminum substrate, where the dashed line is interpolated experimental data [35] [their Fig. 4(b)].

and the rate of contact line dissipation $R_{\mu_{f}}=\int_{\Gamma} \epsilon \mu_{f}(\partial C / \partial t)^{2} d \Gamma . \Omega$ is here the entire volume and $\Gamma$ is the substrate area. In the droplet impact dynamics, we observe that at early times $t^{*}<0.25$ the magnitude of $R_{\rho}^{*}$ decreases rapidly, while $R_{\mu_{\mathrm{f}}}^{*}$ on the other hand increases; see Fig. 6. A minimum in $R_{\rho}^{*}$ and a maximum in $R_{\mu_{\mathrm{f}}}^{*}$ take place at $t^{*} \approx 0.25$, whereas both slowly approach zero as the velocities decrease. Surprisingly, viscous dissipation appears to not play an important role in this regime as both $R_{\mu_{\mathrm{f}}}^{*}$ and $R_{\rho}^{*}$ are much larger for $\beta(t)<\beta_{\max }$.

\section{Scaling law for $\boldsymbol{\beta}_{\max }\left(\mu_{f}\right)$}

Since our simulations fall into both the inertia-viscous and the inertia-capillary regimes, we can further test if our numerical simulations are also consistent with existing scaling laws. We compare our numerical data for $\mu_{f}=0$ [Fig. 7(a)] and the measured $\mu_{f}$ [Fig. 7(b)] with another set of independent experimental data [23] for impacting droplets of different fluids. We use the scaling law 


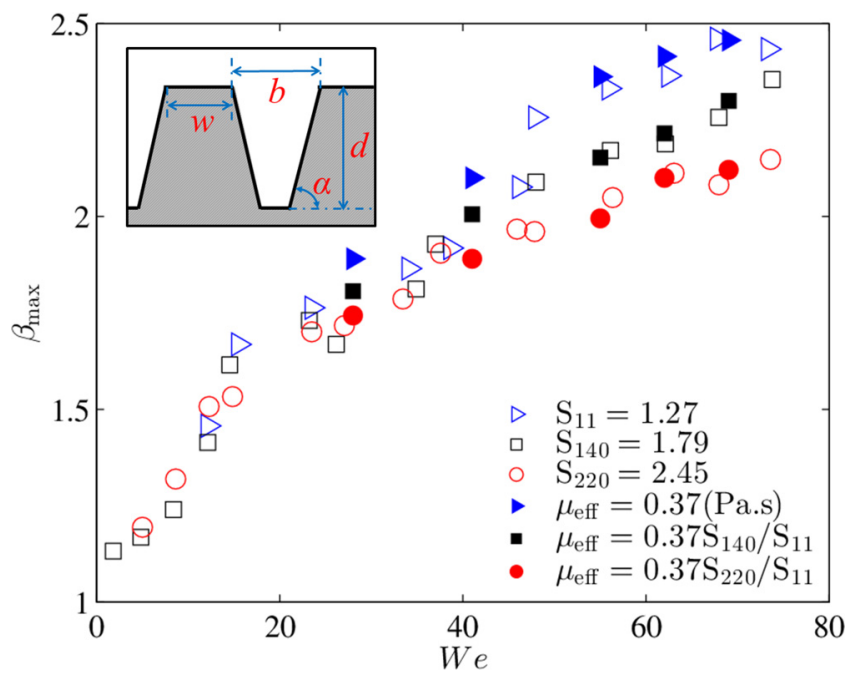

FIG. 5. $\beta_{\max }$ for water droplets impacting onto microtextured stainless steel substrates with grooves along one direction (see inset). The filled markers are numerical simulations with $\mu_{\text {eff }} \sim S \mu_{f}$. The hollow markers are experimental results (Fig. 3(b) in Ref. [34]). The inset shows the geometric parameters $(w, b, d$, and $\alpha$ ) for the grooved substrate (Fig. 1 in Ref. [34]).

for $\beta_{\max }=\operatorname{Re}^{\frac{1}{5}} f\left(\mathrm{WeRe}^{-\frac{2}{5}}\right)$ that couples the inertia-viscous and inertia-capillary regimes [22,23] and is illustrated by the line in Fig. 7. We see in Fig. 7(a) that $\mu_{f}=0$ creates results that deviate from the scaling law and the experiments. One exception is the simulations of water droplets; this is not surprising as the Reynolds number for water is ten times larger than for the glycerol-water mixture and inertial effects are therefore much more dominant. Including the effect of contact line

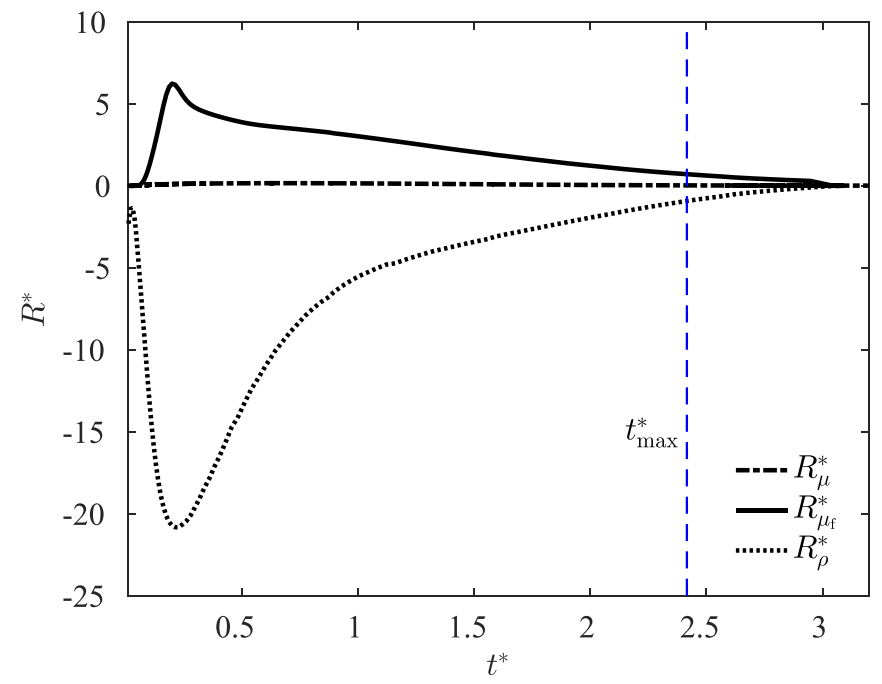

FIG. 6. The nondimensional rate of change of kinetic energy $\left(R_{\rho}^{*}=\int_{\Omega} \frac{1}{2} \operatorname{Ca} \frac{1}{2} \operatorname{Re} \frac{\partial\left[\rho^{*}(C) \mathbf{u}^{* 2}\right]}{\partial t^{*}} d \Omega\right)$, rate of viscous dissipation $\left(R_{\mu}^{*}=\int_{\Omega} \operatorname{Ca} \mu^{*}(C)\left(\nabla \mathbf{u}^{*}+\nabla \mathbf{u}^{* \mathbf{T}}\right): \nabla \mathbf{u}^{*} d \Omega\right)$ and rate of contact line dissipation $\left(R_{\mu_{\mathrm{f}}}^{*}=\right.$ $\left.\int_{\Gamma} D_{w}\left(\frac{\partial C}{\partial t^{*}}\right)^{2} d \Gamma, D_{w}=\mathrm{CaCn} \frac{\mu_{f}}{\mu_{l}}\right)$ for a glycerol-water droplet $(10 \mathrm{mPa} \mathrm{s})$ impacting with; $V_{i}=1 \mathrm{~m} / \mathrm{s}, R=$ $0.92 \mathrm{~mm}$ and $\mu_{f}=0.72 \mathrm{Pas}$, i.e., $\mathrm{Re}=212, \mathrm{Ca}=0.15, \mathrm{We}=31.4 . t_{\max }^{*}=2.41$ is here the time in which the droplet is most deformed along the $r$ direction, $\beta_{\max }=1.83$. 

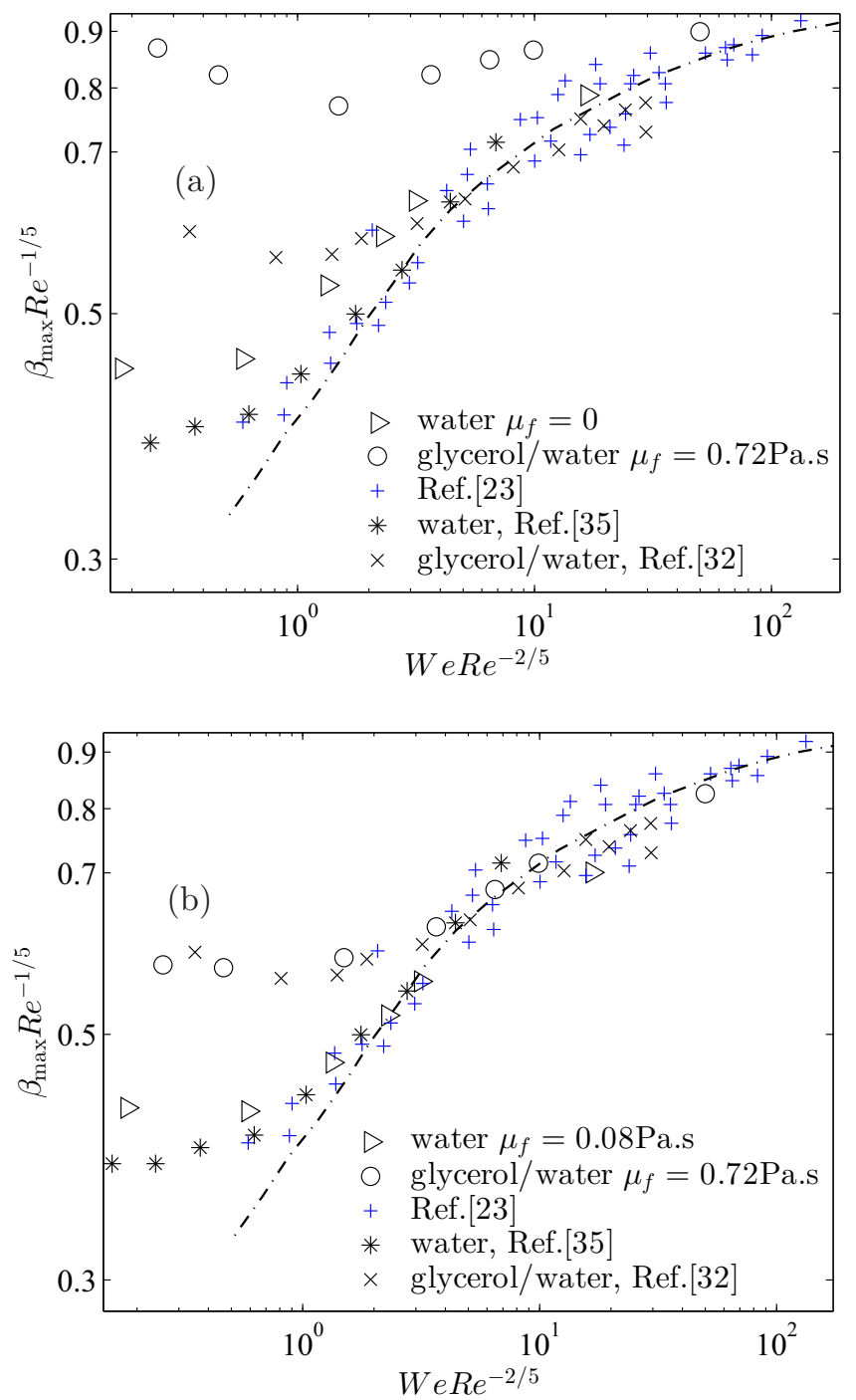

FIG. 7. The maximum spreading factor $\beta_{\max }$ for different liquids, from experiments $[23,32,35]$ (symbols: $+, \times, *)$ and simulations (hollow markers). The dashed line is illustrating [31] the scaling relation $\beta_{\max }=$ $\operatorname{Re}^{\frac{1}{5}} f\left(\mathrm{WeRe}^{-\frac{2}{5}}\right)$. (a) Comparing experiments $[23,32,35]$ with numerical simulations for $\mu_{f}=0$. (b) Comparing experiments $[23,32,35]$ with numerical simulations for $\mu_{f}$ as reported in Table II.

dissipation by using the values for $\mu_{f}$ determined in Figs. 2 and 4 makes the simulated data for $\mathrm{WeRe}^{-\frac{2}{5}}>1$ fit well with the scaling law $\beta_{\max }=\operatorname{Re}^{\frac{1}{5}} f\left(\mathrm{WeRe}^{-\frac{2}{5}}\right)$ and the experiments. However, it is clear that for the regime where the effect from $\mu_{f}$ is expected to be important, i.e., WeRe $\mathrm{R}^{-\frac{2}{5}}<1$, both the experiments and the simulations deviate from the existing scaling law.

To improve the scaling prediction for $\beta_{\max }$ we include the influence from the contact line dissipation into the approximation for the energy balance,

$$
\int_{\Omega} \frac{1}{2} \frac{\partial\left(\rho_{1} \mathbf{u}^{2}\right)}{\partial t} d \Omega \approx \int_{\Omega} \mu_{1}\left(\nabla \mathbf{u}+\nabla \mathbf{u}^{\mathbf{T}}\right): \nabla \mathbf{u} d \Omega+\int_{R} \mu_{f} V_{\mathrm{c}}^{2} d R+\int_{s} \sigma \frac{\partial s}{\partial t} d s
$$



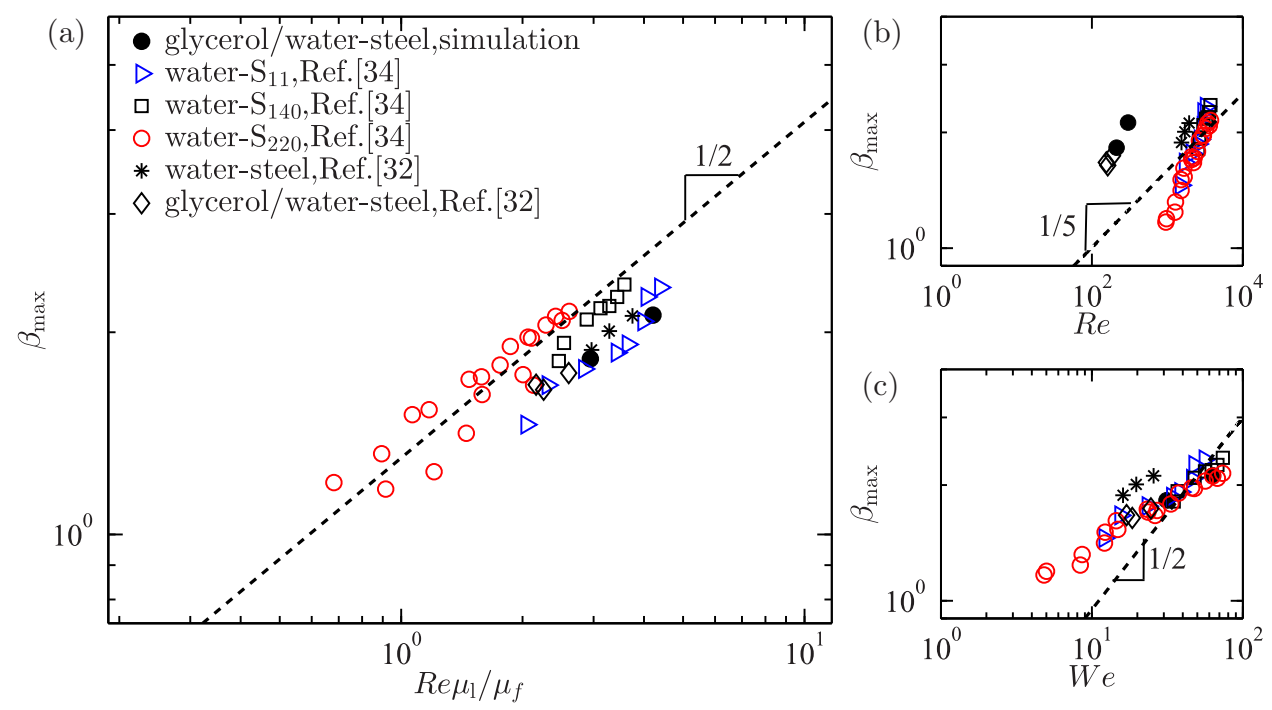

FIG. 8. Scaling droplet impact data on different substrates where $\frac{\mu_{f}}{\mu_{1}}>5\left(\beta_{\max }^{3}+\frac{1}{\mathrm{Ca}}\right)$. (a) $\beta_{\max }$ as a function of $\frac{\mu_{1}}{\mu_{f}} \mathrm{Re}$. Note that the slope of the individual data sets is the same, but has a slightly different prefactor. (b) $\beta_{\max }$ as a function of Re. (c) $\beta_{\max }$ as a function of We. Note that for the grooved substrates $\mathrm{S}_{11}, \mathrm{~S}_{140}$, and $\mathrm{S}_{220}$, we use the measured valued for $\mu_{\text {eff }}$.

with the contact line speed defined as $V_{\mathrm{c}}=\partial r(t) / \partial t$. Note that the contact line dissipation is independent of the interface thickness and can be rewritten [40] as $\int_{\Gamma} \epsilon \mu_{f}(\partial C / \partial t)^{2} d \Gamma=$ $\int_{r_{c}} \mu_{f} V_{\mathrm{c}}^{2} d r_{c}$ with $r_{c}$ as the radial position of the contact line. $s$ is the droplet surface area and the last term on the right-hand side of Eq. (6) is the rate of change of droplet surface energy. Based on the approximated energy balance we assume the following scaling relations [31] for a droplet that has spread to $r_{\max }$ and has a height $h: \mathbf{u}^{2} \sim V_{i}^{2}, \nabla \mathbf{u} \sim V_{i} / h, t \sim r_{\max } / V_{i}, V_{\mathrm{c}} \sim V_{i}, \Omega \sim R^{3}$, $\Gamma \sim r_{\max }$, and $s \sim r_{\max }^{2}$. In addition, mass conservation of the incompressible droplet demands that its volume remains constant and that $R^{3} \sim h r_{\max }^{2}$. By introducing these scaling relations into Eq. (6) we get the following expressions:

$$
\begin{aligned}
\int_{\Omega} \frac{\partial\left(\rho_{\mathrm{l}} \mathbf{u}^{2}\right)}{\partial t} d \Omega & \sim \frac{\rho_{\mathrm{l}} V_{i}^{3} R^{3}}{r_{\max }} \\
\int_{\Omega} \mu_{1}\left(\nabla \mathbf{u}+\nabla \mathbf{u}^{\mathbf{T}}\right): \nabla \mathbf{u} d \Omega & \sim \frac{\mu_{1} V_{i}^{2} r_{\max }^{4}}{R^{3}} \\
\int_{\Gamma} \mu_{f} V_{\mathrm{c}}^{2} d \Gamma & \sim \mu_{f} V_{i}^{2} r_{\max } \\
\int_{s} \sigma \frac{\partial s}{\partial t} d s & \sim \sigma V_{i} r_{\max } .
\end{aligned}
$$

Now substituting these scaling relations into Eq. (6) and rearranging the terms give $\beta_{\max }$ as a function of the contact line friction parameter $\mu_{f}$,

$$
\mathrm{Re}=\beta_{\max }^{2}\left(\frac{\mu_{f}}{\mu_{1}}+\frac{1}{\mathrm{Ca}}+\beta_{\max }^{3}\right) .
$$

Three separate regimes appear, where we immediately see that the two limits $\frac{\mu_{f}}{\mu_{1}}+\frac{1}{\mathrm{Ca}} \ll \beta_{\max }^{3}$ $\left(\beta_{\max } \sim \mathrm{Re}^{\frac{1}{5}}\right)$ and $\frac{\mu_{f}}{\mu_{1}}+\beta_{\max }^{3} \ll \frac{1}{\mathrm{Ca}}\left(\beta_{\max } \sim \mathrm{We}^{\frac{1}{2}}\right)$ recovers the classical scaling laws. However, we identify a new regime where contact line dissipation is a considerable contribution to the energy 
budget, i.e., $\frac{1}{\mathrm{Ca}}+\beta_{\max }^{3} \ll \frac{\mu_{f}}{\mu_{1}}$ with $\beta_{\max } \sim\left(\mu_{1} \operatorname{Re} / \mu_{f}\right)^{\frac{1}{2}}$. To test this new scaling law we plot the experimental data and the numerical simulations for $\mu_{f} / \mu_{1}>5\left(\beta_{\max }^{3}+1 / \mathrm{Ca}\right)$, which follows $\beta_{\max } \sim$ $\left(\operatorname{Re} \mu_{1} / \mu_{f}\right)^{\frac{1}{2}}$, see Fig. 8(a), instead of $\beta_{\max } \sim \mathrm{We}^{\frac{1}{2}}$ in Fig. 8(b) or $\beta_{\max } \sim \operatorname{Re}^{\frac{1}{5}}$ in Fig. 8(c). It would be interesting to experimentally investigate this regime $\left[\mu_{f} / \mu_{1}>5\left(\beta_{\max }^{3}+1 / \mathrm{Ca}\right)\right]$ over a larger parameter space, as the viscosity, surface tension, and impact speed can be controlled and for a known $\mu_{f}$ the effective friction factor can be increased by altering the substrate structure $\left(\mu_{\text {eff }} \sim S \mu_{f}\right)$. Values for $\mu_{f}$ are, in addition to those reported here, already estimated for several different liquidgas-substrate combinations for different viscosities, wettabilities, and substrate roughness $[41,53]$.

\section{CONCLUSIONS}

We have investigated the dynamics of droplets impacting onto solid substrates as a function of their viscosity, substrate wettability, and substrate topography by deploying numerical simulations. By assuming linear response through a Stokes-like drag at the contact line, our simulations rationalize experimental observations for droplet impact on both smooth and rough substrates. Our results highlight that dissipation at the contact line can give a significant contribution to the energy budget and needs to be included to predict the droplet spreading dynamics. We propose a scaling relation for this regime that is dominated by contact line dissipation $\beta_{\max } \sim\left(\frac{\mu_{1}}{\mu_{f}} \operatorname{Re}\right)^{\frac{1}{2}}$, complementing the classical scaling laws for $\beta_{\max }$, i.e., $\beta_{\max } \sim \mathrm{We}^{\frac{1}{2}}$ and $\beta_{\max } \sim \mathrm{Re}^{\frac{1}{5}}$ that are also identified in the numerical simulations. Moreover, our simulations highlight the link between substrate roughness and the effective contact line friction factor $\mu_{\text {eff }}$ that can provide a unifying framework to describe droplet impact dynamics.

\section{ACKNOWLEDGMENTS}

The computations in this paper were run on the Abel Cluster supported by UNINETT Sigma 2 and the Department for Research Computing at the University of Oslo.

[1] R. Dykhuizen, Review of impact and solidification of molten thermal spray droplets, J. Thermal Spray Technol. 3, 351 (1994).

[2] D. Attinger, Z. Zhao, and D. Poulikakos, An experimental study of molten microdroplet surface deposition and solidification: Transient behavior and wetting angle dynamics, J. Heat Transfer 122, 544 (2000).

[3] S. Fathi, P. Dickens, and F. Fouchal, Regimes of droplet train impact on a moving surface in an additive manufacturing process, J. Mater. Process. Technol. 210, 550 (2010).

[4] V. Bergeron, D. Bonn, J. Y. Martin, and L. Vovelle, Controlling droplet deposition with polymer additives, Nature (London) 405, 772 (2000).

[5] N. Z. Mehdizadeh, S. Chandra, and J. Mostaghimi, Formation of fingers around the edges of a drop hitting a metal plate with high velocity, J. Fluid Mech. 510, 353 (2004).

[6] S. Mandre, M. Mani, and M. P. Brenner, Precursors to Splashing of Liquid Droplets on a Solid Surface, Phys. Rev. Lett. 102, 134502 (2009).

[7] L. Duchemin and C. Josserand, Curvature singularity and film-skating during drop impact, Phys. Fluids 23, 091701 (2011).

[8] J. M. Kolinski, S. M. Rubinstein, S. Mandre, M. P. Brenner, D. A. Weitz, and L. Mahadevan, Skating on a Film of Air: Drops Impacting on a Surface, Phys. Rev. Lett. 108, 074503 (2012).

[9] W. Bouwhuis, R. C. A van der Veen, T. Tran, D. L. Keij, K. G. Winkels, I. R. Peters, D. van der Meer, C. Sun, J. H. Snoeijer, and D. Lohse, Maximal Air Bubble Entrainment at Liquid-Drop Impact, Phys. Rev. Lett. 109, 264501 (2012). 
[10] Y. Liu, P. Tan, and L. Xu, Compressible air entrapment in high-speed drop impacts on solid surfaces, J. Fluid Mech. 716, R9 (2013).

[11] C. W. Visser, P. E. Frommhold, S. Wildeman, R. Mettin, D. Lohse, and C. Sun, Dynamics of highspeed micro-drop impact: Numerical simulations and experiments at frame-to-frame times below $100 \mathrm{~ns}$, Soft Matter 11, 1708 (2015).

[12] E. Li and S. T. Thoroddsen, Time-resolved imaging of a compressible air disk under a drop impacting on a solid surface, J. Fluid Mech. 780, 636 (2015).

[13] M. M. Driscoll and S. R. Nagel, Ultrafast Interference Imaging of Air in Splashing Dynamics, Phys. Rev. Lett. 107, 154502 (2011).

[14] K. Yokoi, Numerical studies of droplet splashing on a dry surface: Triggering a splash with the dynamic contact angle, Soft Matter 7, 5120 (2011).

[15] C. W. Visser, Y. Tagawa, C. Sun, and D. Lohse, Microdroplet impact at very high velocity, Soft Matter 8, 10732 (2012).

[16] G. Riboux and J. M. Gordillo, Experiments of Drops Impacting a Smooth Solid Surface: A Model of the Critical Impact Speed for Drop Splashing, Phys. Rev. Lett. 113, 024507 (2014).

[17] L. Xu, W. W. Zhang, and S. R. Nagel, Drop Splashing on a Dry Smooth Surface, Phys. Rev. Lett. 94, 184505 (2005).

[18] A. I. Fedorchenko, A.-B. Wang, and Y.-H. Wang, Effect of capillary and viscous forces on spreading of a liquid drop impinging on a solid surface, Phys. Fluids 17, 093104 (2005).

[19] I. V. Roisman, R. Rioboo, and C. Tropea, Normal impact of a liquid drop on a dry surface: Model for spreading and receding, Proc. Royal Soc. London, Ser. A 458, 1411 (2002).

[20] T. Bennett and D. Poulikakos, Splat-quench solidification: Estimating the maximum spreading of a droplet impacting a solid surface, J. Mater. Sci. 28, 963 (1993).

[21] G. Lagubeau, M. A. Fontelos, C. Josserand, A. Maurel, V. Pagneux, and P. Petitjeans, Spreading dynamics of drop impacts, J. Fluid Mech. 713, 50 (2012).

[22] J. Eggers, M. A. Fontelos, C. Josserand, and S. Zaleski, Drop dynamics after impact on a solid wall: Theory and simulations, Phys. Fluids 22, 062101 (2010).

[23] N. Laan, K. G. de Bruin, D. Bartolo, C. Josserand, and D. Bonn, Maximum Diameter of Impacting Liquid Droplets, Phys. Rev. Appl. 2, 044018 (2014).

[24] B. L. Scheller and D. W. Bousfield, Newtonian drop impact with a solid surface, AIChE J. 41, 1357 (1995).

[25] M. Pasandideh-Fard, Y. Qiao, S. Chandra, and J. Mostaghimi, Capillary effects during droplet impact on a solid surface, Phys. Fluids 8, 650 (1996).

[26] T. Mao, D. Kuhn, and H. Tran, Spread and rebound of liquid droplets upon impact on flat surfaces, AIChE J. 43, 2169 (1997).

[27] S. Chandra and C. Avedisian, On the collision of a droplet with a solid surface, Proc. Royal Soc. London, Ser. A 432, 13 (1991).

[28] D. Vadillo, A. Soucemarianadin, C. Delattre, and D. Roux, Dynamic contact angle effects onto the maximum drop impact spreading on solid surfaces, Phys. Fluids 21, 122002 (2009).

[29] C. Ukiwe and D. Y. Kwok, On the maximum spreading diameter of impacting droplets on well-prepared solid surfaces, Langmuir 21, 666 (2005).

[30] C. Clanet, C. Béguin, D. Richard, and D. Quéré, Maximal deformation of an impacting drop, J. Fluid Mech. 517, 199 (2004).

[31] C. Josserand and S. Thoroddsen, Drop impact on a solid surface, Annu. Rev. Fluid Mech. 48, 365 (2016).

[32] J. Lee, N. Laan, K. de Bruin, G. Skantzaris, N. Shahidzadeh, D. Derome, J. Carmeliet, and D. Bonn, Universal rescaling of drop impact on smooth and rough surfaces, J. Fluid Mech. 786, R4 (2015).

[33] R. Kannan and D. Sivakumar, Drop impact process on a hydrophobic grooved surface, Colloids Surf. A 317, 694 (2008).

[34] V. Vaikuntanathan and D. Sivakumar, Maximum spreading of liquid drops impacting on groove-textured surfaces: Effect of surface texture, Langmuir 32, 2399 (2016).

[35] J. B. Lee, D. Derome, R. Guyer, and J. Carmeliet, Modeling the maximum spreading of liquid droplets impacting wetting and nonwetting surfaces, Langmuir 32, 1299 (2016). 
[36] P. Tsai, S. Pacheco, C. Pirat, L. Lefferts, and D. Lohse, Drop impact upon micro- and nanostructured superhydrophobic surfaces, Langmuir 25, 12293 (2009).

[37] R. C. van der Veen, M. H. Hendrix, T. Tran, C. Sun, P. A. Tsai, and D. Lohse, How microstructures affect air film dynamics prior to drop impact, Soft Matter 10, 3703 (2014).

[38] S. Robson and G. R. Willmott, Asymmetries in the spread of drops impacting on hydrophobic micropillar arrays, Soft Matter 12, 4853 (2016).

[39] A. Yarin, Drop impact dynamics: Splashing, spreading, receding, bouncing, Annu. Rev. Fluid Mech. 38, 159 (2006).

[40] A. Carlson, M. Do-Quang, and G. Amberg, Dissipation in rapid dynamic wetting, J. Fluid Mech. 682, 213 (2011).

[41] A. Carlson, G. Bellani, and G. Amberg, Contact line dissipation in short-time dynamic wetting, Europhys. Lett. 97, 44004 (2012).

[42] D. Jacqmin, Calculation of two-phase Navier-Stokes flows using phase-field modeling, J. Comput. Phys. 155, 96 (1999).

[43] J. W. Cahn and J. E. Hilliard, Free energy of a nonuniform system, I: Interfacial free energy, J. Chem. Phys. 28, 258 (1958).

[44] D. Jacqmin, Contact-line dynamics of a diffuse fluid interface, J. Fluid Mech. 402, 57 (2000).

[45] P. Yue, C. Zhou, and J. J. Feng, Sharp-interface limit of the Cahn-Hilliard model for moving contact lines, J. Fluid Mech. 645, 279 (2010).

[46] F. Magaletti, F. Picano, M. Chinappi, L. Marino, and C. M. Casciola, The sharp-interface limit of the Cahn-Hilliard/Navier-Stokes model for binary fluids, J. Fluid Mech. 714, 95 (2013).

[47] G. Amberg, R. Tönhardt, and C. Winkler, Finite element simulations using symbolic computing, Math. Comp Simul. 49, 257 (1999).

[48] V. Khatavkar, P. Anderson, P. Duineveld, and H. Meijer, Diffuse-interface modeling of droplet impact, J. Fluid Mech. 581, 97 (2007).

[49] Q. Zhang, T.-Z. Qian, and X.-P. Wang, Phase field simulation of a droplet impacting a solid surface, Phys. Fluids 28, 022103 (2016).

[50] Y. Wang, M. Do-Quang, and G. Amberg, Events and conditions in droplet impact: A phase field prediction, Int. J. Multiphase Flow 87, 54 (2016).

[51] W. Villanueva and G. Amberg, Some generic capillary-driven flows, Int. J. Multiphase Flow 32, 1072 (2006).

[52] A. Carlson, G. Bellani, and G. Amberg, Universality in dynamic wetting dominated by contact-line friction, Phys. Rev. E 85, 045302 (2012).

[53] J. Wang, M. Do-Quang, J. J. Cannon, F. Yue, Y. Suzuki, G. Amberg, and J. Shiomi, Surface structure determines dynamic wetting, Sci. Rep. 5, 8474 (2015). 